\title{
Enquête parasitologique et sérologique (Elisa-indirect) sur les trypanosomoses des bovins dans la zone de Sidéradougou, Burkina Faso
}

\author{
M. Desquesnes 1,3* J.F. Michel 1,3 S. de La Rocque 1,3 \\ P. Solano ${ }^{1,2}$ L. Millogo ${ }^{1}$ Z. Bengaly ${ }^{1}$ I. Sidibe ${ }^{1}$
}

\begin{abstract}
Mots-clés
Bovin - Trypanosoma vivax - Trypanosoma brucei - Trypanosoma congolense - Parasitologie - Test Elisa Hématocrite - Morbidité - Burkina Faso.
\end{abstract}

\begin{abstract}
Résumé
U ne enquête parasitologique et sérologique sur les trypanosomoses des bovins a été réalisée dans le secteur nord de la zone de Sidéradougou (Burkina Faso), en novembre et décembre 1997. Un millier de bovins ont été examinés sur la base d'un échantillonnage stratifié aléatoire. L'âge et la race des animaux, la nature et la date du dernier traitement trypanocide ont été enregistrés. Les examens parasitologiques ont été effectués par la méthode du buffy coat, la mesure de l'hématocrite a été recueillie et les tests Elisa-indirect ont été réalisés dans trois systèmes différents, avec les antigènes solubles de Trypanosoma vivax, $\mathrm{T}$. brucei ou $\mathrm{T}$. congolense (type savane). La technique parasitologique a fourni 5,3 p. 100 d'échantillons positifs, dominés par l'espèce T. congolense. Les examens sérologiques ont indiqué une séroprévalence de 81,7 p. 100 $( \pm 2,4 \mathrm{p} .100)$ les trois tests confondus. L'incidence annuelle moyenne a été estimée à 52 p. 100 ( \pm 11 p. 100). Les scores de positivité ont indiqués des séroprévalences par espèce de 79 p. 100 pour T. vivax, 3 p. 100 pour T. brucei, et 28 p. 100 pour T. congolense. Dans cette situation enzootique, la parasitologie est peu sensible, mais, associée à la valeur de I'hématocrite, elle a permis d'estimer l'importance des cas de trypanosomose maladie à environ 15 p. 100. Dans le secteur étudié, les trypanosomoses bovines demeurent une préoccupation majeure. Elles sévissent de manière enzootique avec une prédominance nette des infections par T. vivax, une prévalence et un impact clinique élevés de T. congolense. Les données générées par cette enquête seront intégrées dans un système d'information géographique (Sig) mis en place dans la zone pour l'évaluation du risque trypanosomien.
\end{abstract}

\section{INTRODUCTION}

En Afrique subsaharienne, les trypanosomoses animales sont considérées comme les plus importantes maladies du bétail transmises par des vecteurs. Le contrôle de ces maladies repose sur l'utilisation de trypanocides, l'élevage de bétail trypanotolérant et la lutte contre leurs principaux vecteurs, les glossines, notamment à l'aide de systèmes attractifs toxiques (32). Ces mesures trouvent leur limite dans l'apparition de résistances aux trypanocides et la difficulté à maintenir durablement la pression de lutte. La nécessité

1. Cirdes, BP 454, 01 Bobo-Dioulasso, Burkina Faso

2. Ird, BP 5045, 34032 Montpellier, France

3. Cirad-emvt, Campus de Baillarguet, 34398 Montpellier Cedex 5, France

* Auteur pour la correspondance

Cirad-emvt, Cirdes, BP 454, 01 Bobo-Dioulasso, Burkina Faso

Tél : + 226972287 ; Fax : + 226972320 ; E-mail : m.desquesnes@fasonet.bf est donc apparue d'identifier les lieux à risques dans les secteurs prioritaires de développement, afin de réduire le coût de la lutte et d'en accroître l'efficacité (11).

Au Burkina Faso, la zone agropastorale de Sidéradougou, située au sud-est de Bobo-Dioulasso, est un excellent exemple. Dans les années quatre-vingts, cette zone a été pressentie pour devenir un site d'accueil des élevages transhumants de la région en saison sèche. Elle a par conséquent fait l'objet de prospections entomologiques (8) qui ont induit un plan de lutte. Ce plan reposait sur une alternance saisonnière de pose d'écrans imprégnés d'insecticides et de lâchers de mâles stériles. Deux ans plus tard, les glossines étaient éradiquées de la zone $(9,28,32)$. Malheureusement, le système de barrières n'a pu être maintenu convenablement et, dès 1986, la zone était à nouveau envahie (2). Quinze ans plus tard, une nouvelle prospection entomologique a confirmé la recolonisation du milieu par les glossines riveraines (Glossina palpalis gambiensis et $G$. tachinoides) sur l'ensemble du réseau hydrographique (10). 
Dans le cadre de l'Action thématique programmée Santé-environnement du Cirad-emvt (11), une nouvelle approche de la problématique des trypanosomoses a été instaurée dans le secteur nord de la zone de Sidéradougou, dont le cheptel bovin est estimé à 16500 têtes (29). Cette activité multidisciplinaire regroupe des données entomologiques, parasitologiques (Trypanosoma vivax, T. brucei et $T$. congolense, chez l'hôte et le vecteur), la connaissance du milieu naturel, la répartition des bovins ainsi que les pratiques pastorales et médicales qui peuvent être analysées dans un système d'information géographique $(10,11,12,13)$. La détermination de la prévalence des infections à trypanosomes chez les bovins est une des composantes de ce système d'information.

Une enquête sur les trypanosomoses bovines a donc été réalisée avec l'objectif de déterminer, par des méthodes parasitologiques et sérologiques, les prévalences des principaux trypanosomes pathogènes des bovins. Dans le contexte étudié (présence de T. vivax, $T$. brucei et $T$. congolense), la réalisation de trois réactions sérologiques spécifiques (Elisa) était nécessaire pour obtenir une sensibilité maximale dans la détection des anticorps dirigés contre les trypanosomes pathogènes (26).

\section{MATERIEL ET METHODES}

\section{Echantillonnage, prélèvements et diagnostics parasitologiques}

L'enquête a été réalisée après la saison des pluies, en novembre et décembre 1997. Partant d'un recensement terrestre exhaustif des bovins de la zone effectué en 1997 (29), un millier de bovins ont été sélectionnés selon un échantillonnage stratifié aléatoire (5) basé sur la taille des troupeaux, pour estimer la prévalence dans toute la zone avec une erreur maximale de 5 p. 100. Dans les petits et moyens troupeaux ( $\leq 30$ têtes), les échantillons ont été prélevés sur 14 individus; dans les grands troupeaux (> 30 têtes), 15 à 20 prélèvements individuels ont été réalisés.

Les principaux paramètres intervenant sur le diagnostic et sa signification ont été enregistrés dans un questionnaire : âge, race, état corporel (indice de condition noté de 1 à 5), nature et date du dernier traitement trypanocide, nombre d'animaux.

Les prélèvements ont été effectués à la veine jugulaire des animaux en tube hépariné de $5 \mathrm{ml}$. Les examens parasitologiques ont été réalisés dans les deux heures qui ont suivi le prélèvement. Les plasmas ont été récoltés après centrifugation et conservés à - $20^{\circ} \mathrm{C}$ jusqu'à la réalisation des tests Elisa.

Les examens parasitologiques ont été effectués par la méthode du buffy coat, ou Mbc (observation microscopique du buffy coat extrait de tubes à hématocrite, placé entre lame et lamelle) (31) ; la mesure de l'hématocrite a été enregistrée et les espèces identifiées (4).

\section{Diagnostics sérologiques}

Les examens sérologiques ont été effectués par Elisa-indirect dans trois systèmes avec sensibilisation des plaques par les antigènes solubles $(5 \mu \mathrm{g} / \mathrm{ml})$ de T. vivax (IL1392) (20), T. brucei (ILTat1.2) (7) ou $T$. congolense type savane (IL1180) (20). Le protocole (15) est dérivé de Ferenc et coll. (17) ; il utilise un conjugué commercial anti-bovin (réf. A 5295, Sigma).

Les résultats des test Elisa ont été exprimés en pourcentage de positivité relative (PPRv, PPRb et PPRc) par rapport à des échantillons de référence positifs et négatifs (14). Les seuils de positivité (SP) de chaque test ( $\mathrm{SPv}, \mathrm{SPb}$ et $\mathrm{SPc}$ ) ont été calculés classiquement par la moyenne des PPR de 100 échantillons négatifs (issus d'une zone indemne de trypanosomose) augmentée de trois écarts types. Les PPR obtenus dans les trois systèmes ont été standardisés (les valeurs standardisées ont été notées $\mathrm{V}, \mathrm{B}$ et $\mathrm{C}$ ) sur la base du seuil de positivité du test $T$. brucei selon les relations suivantes :

$$
\begin{aligned}
& \mathrm{V}=\mathrm{PPRv} \times \mathrm{SPb} / \mathrm{SPv} \\
& \mathrm{B}=\mathrm{PPRb} \\
& \mathrm{C}=\mathrm{PPRc} \times \mathrm{SPb} / \mathrm{SPc}
\end{aligned}
$$

Ainsi les trois tests possèdent un seuil de positivité commun dont la valeur est celle du SPb.

\section{Interprétation par le seuil de positivité}

L'interprétation des résultats aux trois tests par le seuil de positivité indique la présence d'anticorps dirigés contre Trypanosoma spp., avec une très bonne sensibilité (26), mais sans permettre de préciser les espèces puisque Elisa-indirect n'est pas spécifique d'espèce (18).

\section{Interprétation des scores maximums de positivité}

Bien que le diagnostic sérologique par recherche d'anticorps ne permette pas de préciser les espèces, les auteurs s'accordent sur le fait que l'intensité des réactions homologues est presque toujours supérieure à celle des réactions hétérologues $(15,16,17,18,23$, $24,30)$. En effet, des plasmas de moutons expérimentalement infectés (infections monospécifiques), testés en Elisa dans les mêmes conditions, fournissent 98,5 p. 100 de réponses homologues supérieures aux réponses hétérologues (Desquesnes et Bengaly, comm. pers.). En conséquence, le score de PPR le plus élevé des trois tests indique avec une très forte probabilité la présence d'anticorps dirigés contre l'un au moins des parasites rencontrés par l'hôte, sans toutefois permettre de détecter les infections mixtes.

Cette méthode d'interprétation a été appliquée à l'ensemble des échantillons. Elle permet une estimation de la prévalence relative des trois espèces recherchées. Sur cette base, un modèle d'extrapolation a été appliqué pour déterminer le taux d'infections mixtes et estimer les séroprévalences des trois Trypanosoma spp. présents dans la population étudiée.

Les liaisons entre résultats parasitologiques et sérologiques, valeur de l'hématocrite, âge, date du dernier traitement trypanocide..., ont été étudiées par des méthodes statistiques classiques.

\section{RESU LTATS}

\section{Echantillonnage, hématocrites et diagnostics parasitologiques}

Au total, 1019 animaux ont été examinés dans 125 troupeaux. La répartition par type de bovins était la suivante : 54 taurins (désignant ici l'ensemble des taurins de type trypanotolérant), 424 métis, 501 zébus et 40 non déterminés.

L'hématocrite moyen observé était de 30,52 p. $100(\mathrm{~s}=5,4)$ : 31,6 p. 100 chez les taurins, 30,0 p. 100 chez les métis, 30,9 p. 100 chez les zébus. Les individus présentant des valeurs de l'hématocrite inférieures à 25 p. 100 (seuil classiquement considéré comme révélateur d'un état pathologique) (1) étaient 125 (12,3 p. 100) dont seulement 3 p. 100 chez les taurins, contre 13 p. 100 chez les métis et 12 p. 100 chez les zébus.

Les examens parasitologiques ont fourni 5,3 p. 100 d'échantillons positifs (54/1019) pour Trypanosoma spp. (trypanosomes pathogènes), dont 19 positifs pour $T$. vivax (1,9 p. 100), 3 pour T. brucei 
(0,3 p. 100) et 35 pour $T$. congolense (3,4 p. 100), répartis comme indiqué dans le tableau I. L'importance relative des trois espèces apparaît entre parenthèses dans le tableau II. L'espèce prédominante était T. congolense (65 p. 100 des cas).

\section{Résultats des tests Elisa par le seuil de positivité}

Les 1019 échantillons de plasma testés en Elisa-indirect ont fourni 80,5 p. 100 de résultats positifs pour T. vivax, 50 p. 100 pour T. brucei et 45 p. 100 pour $T$. congolense. La séroprévalence des animaux porteurs d'anticorps dirigés contre Trypanosoma spp. (les trois tests confondus) était de 81,7 p. 100 ( $\pm 2,4$ p. 100). La répartition des résultats par système est indiquée dans le tableau I. Seul le test Elisa $T$. vivax a fourni un nombre important de résultats monospécifiques (222 échantillons positifs), indiquant une forte prédominance des infections par cette espèce.

\section{Résultats des tests Elisa par le score maximum de positivité}

Sur 832 échantillons positifs à l'un au moins des trois tests, les scores de PPR les plus élevés indiquaient $12 \mathrm{~T}$. brucei, $154 T$. congolense et $666 T$. vivax. Ces résultats montrent, avec une forte probabilité, au moins une espèce de trypanosome infectant ou ayant infecté l'individu. Ils sous-estiment les prévalences réelles de chaque parasite puisqu'ils ne tiennent pas compte des infections mixtes, mais ils permettent de calculer les séroprévalences relatives des trois parasites

En appliquant ces proportions relatives à l'ensemble des échantillons positifs, les effectifs théoriques des infections mixtes ont été calculés. Par exemple, parmi les 666 échantillons indiquant une infection à T. vivax, 18,5 p. 100 auraient été également infectés par T. congolense et 1,5 p. 100 par T. brucei. En procédant ainsi pour chacun des effectifs précédents, on a obtenu les séroprévalences estimées sur la totalité de l'échantillonnage (tableau II). Avec ce mode d'interprétation, la séroprevalence s'élevait à 79 p. 100 pour T. vivax , 3 p. 100 pour T. brucei et 28 p. 100 pour T. congolense.

\section{Variations selon l'âge}

L'âge moyen des animaux examinés $(\mathrm{n}=1010)$ était de 4,3 ans ( $\mathrm{s}=3,1)$. Les animaux séronégatifs $(\mathrm{n}=187)$ avaient un âge moyen de 1,6 an $(\mathrm{s}=1,5)$, les séropositifs $(\mathrm{n}=823)$, de 4,9 ans $(\mathrm{s}=3,0)$, les triples positifs $(\mathrm{n}=348)$ de 5,4 ans $(\mathrm{s}=2,9)$. Toutes ces moyennes sont significativement différentes $(\mathrm{p}<0,01)$ et indiquent l'augmentation graduelle de la séroprévalence avec l'âge.

Les individus ont été regroupés en 9 classes d'âge dont les limites, les effectifs et les séroprévalences sont indiqués dans la figure 1.

Avec le test Elisa $T$. vivax, la séroprévalence observée chez les veaux de moins de 4 mois $(n=39)$ était de 31 p. 100 ( \pm 14 p. 100). Tous les animaux positifs en sérologie étaient négatifs en parasitologie. Ces échantillons n'étaient positifs qu'avec le test Elisa T. vivax. Dans la classe d'âge suivante, la séroprévalence était stable puis elle croissait régulièrement et rapidement de 30 à 85 p. 100 entre l'âge de 10 mois et celui de 3 ans, pour atteindre un plateau voisin de 95 p. 100 vers 4 ans.

Avec le test Elisa T. brucei, on n'a pas détecté pas d'anticorps chez les veaux de moins de 4 mois. La séroprévalence croissait régulièrement de 0 p. 100 à 50 p. 100 entre 4 mois et 3 ans, pour atteindre un plateau voisin de 60 p. 100 vers 4 ans.

Avec le test Elisa $T$. congolense, on n'a détecté que 2 animaux positifs dans la classe d'âge inférieur à 4 mois. La séroprévalence croissait régulièrement de 5 à 65 p. 100 entre 0 et 9 ans sans former véritablement de plateau.

A l'échelle de la population échantillonnée, une estimation de l'incidence annuelle moyenne des infections par les trypanosomes (toutes espèces confondues) a pu être faite sur la base de la séroprévalence observée chez les animaux de 10 à 12 mois $(\mathrm{n}=77)$; elle était de 52 p. 100 ( \pm 11 p. 100). L'étude des scores maximums de positivité et le calcul des effectifs théoriques des infections mixtes dans cette classe d'âge ont permis d'estimer l'incidence annuelle par espèce à $51 \mathrm{p}$. 100 pour T. vivax, $14 \mathrm{p} .100$ pour T. congolense et 2,5 p. 100 pour T. brucei.

\section{Prévalences relatives des trypanosomes}

Selon la technique de détection utilisée, les prévalences et séroprévalences relatives observées ou estimées ont été rapportées dans le tableau II. Si les examens parasitologiques montraient que T. congolense était l'espèce dominante (65 p. 100), l'estimation par la sérologie indiquait T. vivax (80 p. 100). Les prévalences relatives observées par la parasitologie et estimées par la sérologie ne condordaient que pour l'espèce T. brucei.

Tableau I

Résultats parasitologiques et sérologiques (par le seuil de positivité)

\begin{tabular}{lccrr} 
& \multicolumn{2}{c}{ Examens parasitologiques } & \multicolumn{2}{c}{ Examens sérologiques } \\
\cline { 2 - 4 } Tests positifs & Pourcentage observé & Effectifs & Pourcentage observé & Effectifs \\
\hline Tv $*$ seul & $1,7 \%$ & 17 & $21,8 \%$ & 222 \\
Tb ** seul & $0,1 \%$ & 1 & $0,1 \%$ & 1 \\
Tc *** seul & $3,2 \%$ & 33 & $1,1 \%$ & 11 \\
Tv et Tb & $0,1 \%$ & 1 & $15,1 \%$ & 154 \\
Tv et Tc & $0,1 \%$ & 1 & $9,1 \%$ & 93 \\
Tb et Tc & $0,1 \%$ & 1 & $0 \%$ & 0 \\
Tv, Tb et Tc & $0,0 \%$ & 0 & $34,5 \%$ & 351 \\
Trypanosoma spp. & $5,3 \%$ & 54 & $81,7 \%$ & 832 \\
Tests négatifs & $94,7 \%$ & 965 & $18,3 \%$ & 187 \\
Total & $100 \%$ & 1019 & $100 \%$ & 1019
\end{tabular}

*Trypanosoma vivax; ${ }^{* *}$ T. brucei ; *** T. congolense 


\section{Tableau II}

Prévalences et prévalences relatives des Trypanosoma spp. observées et estimées sur 1019 échantillons testés

\begin{tabular}{lrrr} 
Trypanosoma spp & Parasitologie observée * & $\begin{array}{c}\text { Sérologie observée par } \\
\text { le seuil de positivité ** }\end{array}$ & $\begin{array}{c}\text { Sérologie estimée par } \\
\text { les scores de positivité ** }\end{array}$ \\
\hline T. vivax & $1,9 \%(35 \%)$ & $80,5 \%(98,5 \%)$ & $79 \%(80 \%)$ \\
T. brucei & $0,3 \%(5,5 \%)$ & $50 \%(61 \%)$ & $3 \%(1,5 \%)$ \\
T. congolense & $3,4 \%(65 \%)$ & $45 \%(55 \%)$ & $28 \%(18,5 \%)$
\end{tabular}

Entre parenthèses, les prévalences relatives parmi les échantillons positifs

* Prévalences relatives calculées sur un effectif de 54 positifs

*** Prévalences relatives calculées sur un effectif de 832 positifs

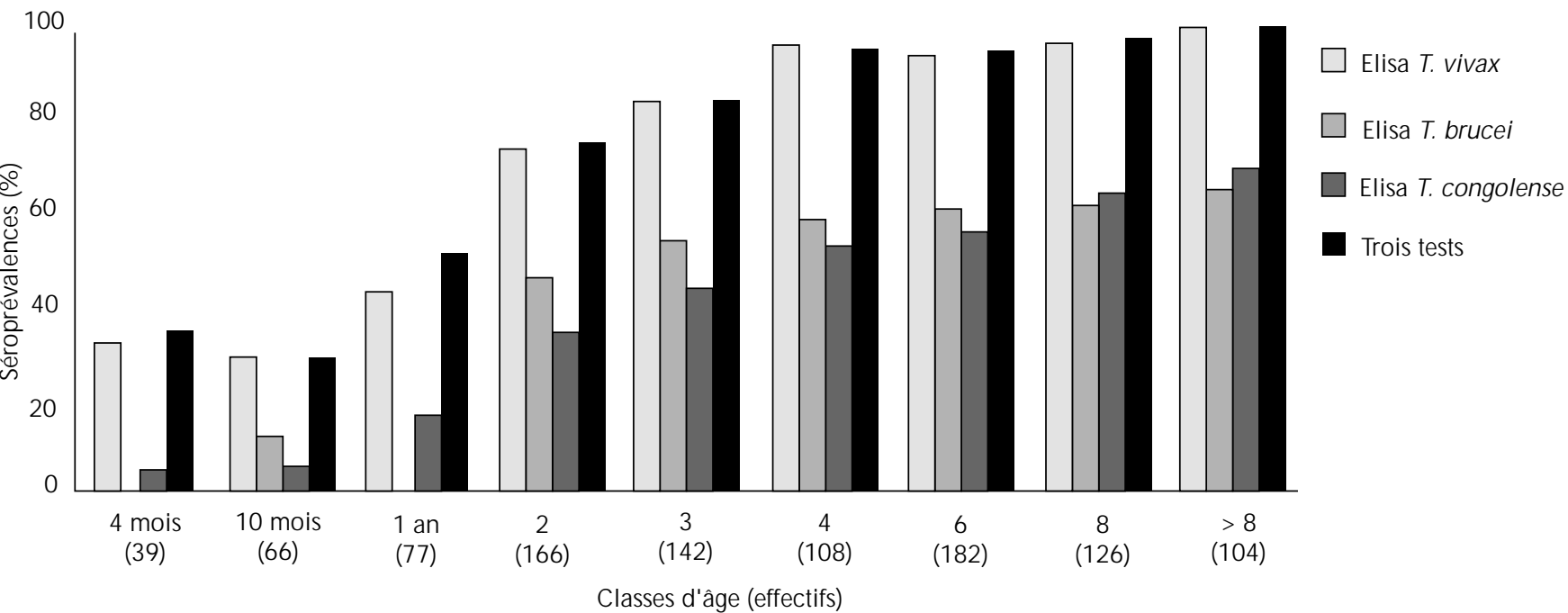

Figure 1 : séroprévalences observées par classe d'âge, avec les Elisa T. vivax, T. brucei, T. congolense et tous tests confondus.

\section{Liaisons à la valeur de l'hématocrite \\ Parasitologie}

Les animaux positifs en parasitologie $(\mathrm{n}=54)$ présentaient un hématocrite moyen de 25,8 p. $100(\mathrm{~s}=5,4)$, alors que celui des animaux négatifs $(\mathrm{n}=956)$ était de 30,8 p. $100(\mathrm{~s}=5,3)$; cette différence est hautement significative $(\mathrm{p}<0,001)$.

Chez les animaux présentant un hématocrite inférieur à 25 (n = 125), la prévalence parasitologique était de 20,8 p. $100(\mathrm{~s}=0,4)$, dont 18 p. 100 pour $T$. congolense et 3,2 p. 100 pour T. vivax. Elle était de 3 p. $100(n=894, \mathrm{~s}=0,17)$ chez les animaux présentant un hématocrite supérieur ou égal à 25 , dont 1,4 p. 100 pour T. congolense et 1,7 p. 100 pour T. vivax. Ces différences sont hautement significatives $(p<0,001)$; elles indiquent que dans le premier groupe les examens parasitologiques étaient plus fréquemment positifs, avec une nette prédominance de $T$. congolense qui n'était pas observée dans le second groupe, moins fréquemment positif.

\section{Sérologie}

Les hématocrites moyens des animaux positifs ou négatifs en sérologie n'étaient pas significativement différents de l'hématocrite moyen de l'ensemble de la population échantillonnée. Néanmoins, chez les animaux présentant un hématocrite inférieur à 25 p. 100 ( $\mathrm{n}=125)$, la séroprévalence de $T$. congolense était de 58,4 p. 100 $(\mathrm{s}=0,49)$. Ce taux était faiblement mais significativement supérieur $(\mathrm{p}<0,03)$ à celui observée chez des animaux présentant un hématocrite supérieur à 25, qui était de 42,7 p. 100 (n = 894, $\mathrm{s}=0,49$ ). En revanche, aucune différence significative n'a été observée pour les séroprévalences de $T$. vivax (voisine de 80,5 p. 100) et T. brucei (voisine de 52 p. 100).

L'interprétation par les scores maximums de positivité indiquait 29 p. 100 de scores en faveur de $T$. congolense chez les animaux présentant un hématocrite inférieur à 25 . Chez les animaux présentant un hématocrite supérieur ou égal à 25 , ils étaient seulement 17 p. 100 (différence significative, $\mathrm{p}<0,001$ ).

Sérologie et parasitologie concordent et indiquent que $T$. congolense était d'avantage présent chez les animaux possédant un hématocrite faible.

\section{Type de bovins}

La valeur de l'hématocrite moyen était significativement plus faible ( $\mathrm{p}<0,001)$ chez les métis $(24,5 \mathrm{p}$. 100) et les zébus (26,2 p. 100) positifs en parasitologie que chez les animaux négatifs $(30,4$ p. 100 et 31,0 p. 100). Chez les taurins trypanotolérants l'hématocrite moyen n'était pas différent selon les résultats de parasitologie ; il était significativement supérieur (voisin de 31,5 p. 100) à celui des autres types de bovins. 
En aucun cas la valeur moyenne de l'hématocrite n'était modifiée selon les résultats de la sérologie.

Il n'a pas été trouvé de différence significative de l'état estimé des animaux (indice de condition) selon la présence de parasites détectables dans le sang et/ou la réponse sérologique.

\section{Liaisons à la nature et la date des traitements trypanocides}

Selon les trois types de traitements déclarés appliqués aux animaux (acéturate de diminazène, chlorure d'isométamidium, ou alternance des deux produits), il n'y avait pas de différence significative entre les prévalences et séroprévalences globales observées ou estimées.

L'étude des résultats individuels selon la date et la nature du dernier traitement trypanocide a fourni les résultats suivants :

- chez les animaux traités depuis trois mois et plus $(\mathrm{n}=566)$, la prévalence parasitologique moyenne était de 4,8 p. 100 ; aucune différence significative n'a été observée selon la nature du traitement (trypanocide ou trypanopréventif) ;

- chez les animaux traités depuis moins de 31 jours $(n=248)$ et depuis 31 à 61 jours $(n=156)$, les prévalences parasitologiques étaient respectivement de 4,8 p. 100 et 9,6 p. 100 ; chez les animaux traité au chlorure d'isométamidium, la prévalence parasitologique était nulle dans la première période, mais de 12,5 p. 100 dans la deuxième, alors que chez les animaux traités à l'acéturate de diminazène, la prévalence était voisine de 7,5 p. 100 quelle que soit la date du dernier traitement.

Certains propriétaires de grands troupeaux ont la capacité financière et une connaissance suffisante de la trypanosomose pour entreprendre des traitements collectifs. Les effectifs, les prévalences parasitologiques et les séroprévalences observées en fonction de la date du dernier traitement déclaré, chez les animaux traités à l'acéturate de diminazène $(\mathrm{n}=305)$, sont présentés dans le tableau III. Alors que l'étude parasitologique ne montrait pas de différence significative des prévalences observées selon la date du dernier traitement, on a observé une chute des séroprévalences dans la catégorie des animaux traités depuis 1 à 2 mois, (différences significatives à des risques inférieurs à 2 p. 100 pour la séroprévalence globale et les séroprévalences de $T$. vivax et $T$. congolense). Cet infléchissement ne se maintenait pas dans la catégorie d'animaux traités depuis plus de 2 mois. Chez les animaux traités au chlorure d'isométamidium on n'a pas observé de variation de séroprévalence selon la date du dernier traitement.

\section{DISCUSSION}

\section{Parasitologie}

Les examens parasitologiques n'ont révélé que 5,3 p. 100 d'échantillons positifs, dominés par l'espèce $T$. congolense, dans un secteur où la trypanosomose est considérée comme la principale maladie vectorielle et où les études chez les vecteurs indiquent de forts niveaux d'infection $(10,12)$. Une étude menée en 1986-1987 révélait des prévalences parasitologiques assez voisines, mais dominées alors par l'espèce $T$. vivax (2). Dans tous les cas, les prévalences parasitologiques étaient très basses. Les techniques parasitologiques ne sont pas adaptées pour l'évaluation du taux d'infection des bovins en secteur hautement enzootique ; de telles observations sont fréquemment rapportées $(18,37)$.

Un sous-échantillonnage représentatif $(\mathrm{n}=258)$, testé par amplification en chaîne par polymérase (ou Polymerase Chain Reaction, $P c r)$ avec les amorces spécifiques des trois parasites étudiés (27), a indiqué une prévalence des trypanosomes pathogènes de 11,6 p. 100, - 2,5 fois supérieure à celle décelée par la technique parasitologique dans le même sous-échantillonnage (35). La Pcr présente un progrès notable dans la spécificité et la sensibilité de la détection des parasites (ou de leur matériel génétique), mais le niveau de parasitémie des animaux reste probablement dans de nombreux cas en-dessous de son seuil de détection. Pour T. brucei et $T$. vivax, l'existence de refuges extra-vasculaires des parasites peut également engendrer des résultats négatifs en $P c r$ malgré un portage effectif $(21,38)$. L'examen exhaustif des échantillons par $P c r$ n'a pu être effectué pour des raisons économiques, mais l'extrapolation des résultats obtenus en $P c r$ à l'ensemble de l'échantillonnage indiquait une prévalence voisine de 15 p. 100.

Sept échantillons étaient positifs en parasitologie et négatifs en sérologie. Ces échantillons provenaient d'animaux jeunes pour la plupart (1,6 ans d'âge moyen), probablement récemment infectés, chez lesquels la séroconversion n'avait pas encore eu lieu.

\section{Sérologie}

Le diagnostic sérologique par recherche d'anticorps n'est pas spécifique d'espèce, de fortes réactions croisées sont observées quel que soit le système de détection utilisé (hémagglutination, Ifi, Elisa...) (18). Les résultats des test Elisa indiqués par les seuils de positivité n'ont donc pas de valeur individuelle spécifique d'espèce, ils signifient que les animaux sont porteurs d'anticorps dirigés contre des trypanosomes pathogènes $(15,17,18,25,26)$.

\section{Tableau III}

Effectifs, prévalences parasitologiques $(\mathrm{Mbc}$ ) et séroprevalences (Elisa) selon la date du dernier traitement à l'acéturate de diminazène dans les grands troupeaux

\begin{tabular}{lcccccc}
$\begin{array}{l}\text { Dernier traitement } \\
\text { (en mois) }\end{array}$ & Effectifs & Mbc * & $\begin{array}{c}\text { Elisa } \\
\text { T. vivax ** }\end{array}$ & $\begin{array}{c}\text { Elisa } \\
\text { T. brucei ** }\end{array}$ & $\begin{array}{c}\text { Elisa } \\
\text { T. congo. ** }\end{array}$ \\
\hline$\leq 1$ & 54 & 3,7 & 76 & 39 & 30 & 76 \\
Tryp. sp. **
\end{tabular}

Mbc : méthode du buffy coat ; * Prévalences en pourcentages ; ** Séroprévalences en pourcentages

En gras, les infléchissements significatifs $(\mathrm{p}<0.02)$ par rapport à la classe précédente ou suivante 
La réalisation simultanée des trois réactions Elisa fournit une sensibilité maximale pour la détection des anticorps dirigés contre les trypanosomes pathogènes (26). La séroprévalence globale, tous tests confondus, était de 81,7 p. 100 , avec un score de 100 p. 100 chez les animaux de plus de 9 ans $(n=104)$. L'âge des animaux pouvant intervenir sur le bruit de fond des réponses sérologiques (19), l'application à cette classe d'âge d'un seuil de positivité augmenté de 25 p. 100 a été réalisée ; elle n'a ramené la séroprévalence qu'à 97 p. 100 au lieu de 100 p. 100. La séroprévalence très élevée chez les animaux âgés ne serait donc pas due à une augmentation du bruit de fond mais bien à un contact avec les trypanosomes. Elle confirme la forte pression parasitaire dans le secteur étudié.

La situation enzootique des trypanosomoses dans la zone de Sidéradougou, appréhendée par les études épidémiologiques antérieures (10), a été confirmée par la sérologie.

Les animaux positifs au test $T$. vivax (80,5 p. 100) représentaient la quasi totalité des animaux positifs à l'un au moins des trois tests (81,7 p. 100), indiquant que les contacts avec $T$. vivax étaient nettement prédominants. Cette observation a été confirmée par l'étude du score le plus élevé aux trois tests (666 scores en faveur de T. vivax sur 832 échantillons positifs).

Les croisements sérologiques du test Elisa $T$. congolense avec les anticorps dirigés contre T. vivax sont moins importants (Desquesnes et Bengaly, comm. pers.). Toutefois Hopkins et coll. (22) utilisent les antigènes de $T$. congolense pour la détection des trois sous-genres pathogènes avec une sensibilité de 88 p. 100. Dans notre enquête, la prévalence des infections à $T$. congolense indiquée par le seuil de positivité était de 45 p. 100, alors que la prévalence estimée (154 scores en faveur de $T$. congolense) n'était que de 28 p. 100. Cette différence est probablement due aux réactions croisées avec $T$. vivax dont la prévalence était très élevée.

Selon le seuil de positivité, la séroprévalence de T. brucei aurait été voisine de 50 p. 100 . Compte tenu des très fortes réactions croisées entre les antigènes des Trypanozoon et les anticorps dirigés contre $T$. vivax et $T$. congolense $(6,16,23,33,34)$, la majeure partie des résultats positifs au test Elisa $T$. brucei peut être attribuée à des réactions croisées. En effet, l'étude des scores les plus élevés dans les trois systèmes indique une très faible prévalence de T. brucei, estimée à moins de 3 p. 100.

Les estimations de séroprévalences qui ont été faites à l'aide des scores de positivité en Elisa fournissent une meilleure approche de la séroprévalence des trois espèces que la seule lecture des résultats par rapport au seuil de positivité.

\section{Etude par classe d'âge}

Chez les animaux de moins de 4 mois, les sérologies positives étaient probablement dues à des anticorps maternels plutôt qu'à l'existence d'infections précoces par T. vivax qui auraient été accompagnées de parasitémies décelables chez ces jeunes animaux. En outre, ces animaux n'étaient positifs que dans le système T. vivax, ce qui confirme la prédominance de ce parasite et indique que les anticorps maternels sont spécifiques d'espèce (pas de réaction croisée avec Elisa T. brucei et Elisa T. congolense). La séroprévalence de $T$. vivax dans la classe 4 à 10 mois $(n=66)$ était stable $(30 \pm 11$ p. 100$)$, ce qui traduit probablement l'élimination des anticorps maternels par une partie de la population et l'acquisition d'anticorps post-infectieux par l'autre partie, infectée précocément.

Dans l'ensemble, la croissance régulière des séroprévalences avec l'âge indique une pression parasitaire relativement constante d'une année à l'autre.

\section{Proportions relatives des trois espèces}

La mise en évidence d'infections actives (Mbc et $P c r$ ) indique une prépondérence de l'espèce $T$. congolense. Les enquêtes parasitologiques menées chez les bovins après la campagne de lutte montraient que l'éradication des glossines avait permis d'éliminer l'espèce $T$. congolense pendant quelques années, durant lesquelles l'espèce $T$. vivax avait dominé (2). Les résultats de la présente enquête indiquent un retour à la domination de l'espèce $T$. congolense.

Bien que la présence de $T$. congolense ait été dominante en parasitologie et par $P c r$, la sérologie indiquait la prépondérance de T. vivax.

L'état hautement enzootique des infections à $T$. vivax, révélé par la sérologie, engendre une situation où les infections sont bien contrôlées par les animaux. Elle se traduit fréquemment par l'impossibilité de diagnostiquer l'infection au moyen de l'examen parasitologique. La situation plus instable de $T$. congolense est en revanche favorable au développement de parasitémies plus importantes, détectables par la parasitologie (ou la Pcr). En outre, en particulier en Afrique occidentale, la virulence de T. congolense est notablement plus forte que celle de T. vivax (36). De ce fait, les parasitémies à $T$. congolense chez les bovins sont souvent importantes, alors que dans les infections chroniques à $T$. vivax les parasites sont souvent indétectables.

Ces éléments concordent et expliquent la différence de prévalences relatives des parasites selon la technique de diagnostic utilisée. Les techniques de détection des parasites (Mbc et $\mathrm{Pcr}$ ) ont permis de détecter les animaux récemment infectés et/ou ne contrôlant que partiellement leur parasitémie (5,3 p. 100 dont 35 p. 100 dues à T. vivax et 65 p. 100 à $T$. congolense), alors que les techniques sérologiques ont détecté les infections dès la deuxième ou troisième semaine quel qu'ait été l'état de portage $(81$ p. 100 dont 79 p. 100 dues à $T$. vivax et 28 p. 100 à T. congolense) .

L'apparente discordance des prévalences relatives observées entre parasitologie et $P c r$ d'une part et sérologie de l'autre était donc probablement liée à la différence de sensibilité des techniques et à la différence d'émergence numérique des parasites dans le sang.

Quoiqu'il en soit, l'émergence de T. congolense, en parasitologie et $P c r$, plus forte que celle de $T$. vivax, a révélé l'impact clinique de la trypanosomose à $T$. congolense (en liaison avec l'effondrement de la valeur de l'hématocrite). La sérologie a confirmé la situation instable des infections à $T$. congolense - et donc leur possible impact sur la production - et a indiqué la prévalence dominante des infections à $T$. vivax. Ces résultats ne sont nullement incompatibles, leur signification épidémiologique et médicale est différente.

Pour T. brucei, la prévalence indiquée par la parasitologie et celle estimée par la sérologie concordaient et étaient toutes deux inférieures à 3 p. 100. Toutefois, la fiabilité des examens sérologiques est plus faible que pour $T$. vivax et $T$. congolense, du fait d'importantes réactions croisées des antigènes de $T$. brucei avec les autres parasites étudiés $(16,34)$.

\section{Estimation du taux d'infections actives}

L'estimation du taux d'infections actives par la parasitologie est efficace dans les foyers épizootiques, où la plupart des animaux, récemment infectés, présentent des parasitémies élevées et détectables. En situation enzootique, sauf stress particulier, une grande partie des animaux activement infectés restent négatifs aux examens parasitologiques (37), particulièrement pour $T$. vivax et $T$. brucei, mais aussi pour $T$. congolense $(18,21)$. Ces phases 
latentes - dites aparasitémiques — sont également observées lors d'infections expérimentales $(15,38)$. Les taux d'infections actives dans ce secteur enzootique étaient donc considérablement sousestimés par les examens parasitologiques.

De même, la sérologie peut sous-estimer la prévalence des animaux activement infectés, du fait du délai de séroconversion positive après infection, évalué à une ou deux semaines $(15,17)$. Toutefois, les animaux subissant une primo-infection sont souvent parasitémiques. Dans notre enquête, le taux d'animaux parasitémiques et séronégatifs était très faible (7 individus) ; la sous-estimation des infections actives était donc négligeable.

A l'inverse, la persistance des anticorps après traitement curatif ou self-cure peut provoquer une surestimation de la prévalence des animaux activement infectés. Il est difficile de mesurer l'importance numérique de ces cas sur le terrain, du fait des incertitudes sur l'application et l'efficacité des traitements et de l'existence de réinfections après ces traitements. La persistance des anticorps détectés par la méthode Elisa est estimée à deux à trois mois (3, $15,17,25)$; elle pourrait être plus longue chez les animaux âgés pour lesquels des seuils de positivité plus élevés sont recommandés (19).

L'étude des séroprévalences dans les groupes d'animaux constitués selon la date du dernier traitement à l'acéturate de diminazène (tableau III) indique qu'une chute de séroprévalence a été enregistrée dans la classe des animaux traités depuis un à deux mois, ce qui correspond bien à la cinétique d'élimination des anticorps après traitement. Toutefois, dans la classe suivante, cet infléchissement ne se maintenait pas et la séroprévalence revenait à une valeur proche de la moyenne générale chez les animaux traités depuis plus de deux mois. Cela tend à montrer que les séroconversions négatives attendues à la suite des traitements sont effacées par des réinfections rapides et/ou par la résurgence des infections à la suite de traitements non stérilisants.

Ces observations sont corroborées par les résultats parasitologiques. Chez les animaux traités depuis trois mois et plus, le taux de positifs était de 4,8 p. 100, égal à celui observé chez les animaux traités depuis moins d'un mois et même inférieur à celui observé chez les animaux traités depuis un à trois mois. Ces chiffres confirment des réinfections rapides et/ou l'inefficacité des traitements. Ils n'indiquent pas que le traitement modifie sensiblement le taux de portage des parasites.

Chez les animaux traités au chlorure d'isométamidium, l'absence d'infléchissement de la sérologie au deuxième mois suivant le traitement et le choix du trypanopréventif traduisaient une pression vectorielle élevée. Cette pression a été confirmée par la détection de cas positifs en parasitologie dès le deuxième mois qui suivait l'usage du trypanopréventif. Elle a révélé en outre une efficacité anormalement faible du produit.

Dans tous les cas, l'inefficacité apparente des traitements pouvait être due à une anamnèse biaisée, à un dosage insuffisant des trypanocides par les éleveurs, à l'utilisation de produits inefficaces (produits frauduleux sans activité trypanocide), à l'existence de refuge extra-vasculaire des parasites ou enfin à leur chimiorésistance.

Compte tenu de ces diverses observations, la prévalence des infections actives restait probablement voisine de la séroprévalence estimée. La faiblesse et la brièveté de l'infléchissement de la séroprévalence dans les classes d'animaux traités récemment ne pouvait se justifier seulement par l'incidence des réinfections. L'efficacité des traitements trypanocides est mise en doute par ces résultats, elle devra être évaluée par des études complémentaires sur les méthodes de traitement (origine et nature des médicaments, doses réelles appliquées), mais également par l'étude de la sensibi- lité des souches locales aux trypanocides.

Quel que soit le taux d'animaux porteurs, la pression parasitaire à l'échelle d'une zone d'élevage est mieux évaluée par le taux de porteurs d'anticorps que par celui des porteurs de parasites. En effet, ce dernier dépend étroitement de la sensibilité de la technique de diagnostic utilisée, de l'état de trypanotolérance de la population étudiée, ainsi que de la fréquence, l'ancienneté et l'efficacité des traitements trypanocides.

\section{Estimation des états pathologiques dus aux trypanosomoses}

Quoique multifactoriel (trypanosomoses, helminthoses, anaplasmose, état nutritionnel...), l'effondrement de la valeur de l'hématocrite est classiquement considéré comme un signe indicateur de trypanosomose maladie (1).

Parmi les animaux, 12,3 p. 100 présentaient des hématocrites inférieurs à 25. Les prévalences détectées chez eux par microscopie et par $P c r$ étaient plus élevées ( 7 fois et 5 fois) que chez les animaux présentant un hématocrite supérieur ou égal à 25 . La sérologie indiquait aussi une séroprévalence supérieure. Dans les deux types d'analyses, $T$. congolense était prédominant.

Chez les métis et les zébus la valeur de l'hématocrite était significativement réduite en présence de parasites décelables dans le sang. L'hématocrite des taurins trypanotolérants n'était pas modifié par la présence ou l'absence de parasite ; il était en outre toujours supérieur à celui des autres types de bovins.

Chez les zébus et les métis, des liaisons fortes existaient entre l'état pathologique, indiqué par une valeur faible de l'hématocrite, la présence de trypanosomes ( $T$. congolense surtout) détectables par la parasitologie ou la $P c r$ et la présence dominante d'anticorps dirigés contre $T$. congolense. Elles indiquaient que 10 à 15 p. 100 des animaux étaient cliniquement affectés par la trypanosomose au moment de l'enquête, plus probablement par des infections à T. congolense. Ces observations n'ont pas été pas vérifiées chez les animaux trypanotolérants.

\section{- CON CLUSION}

Les prévalences révélées par la parasitologie sous-estiment considérablement les taux réels d'infections. La méthode du buffy coat est mal adaptée à l'estimation de la prévalence des infections ou de la pression parasitaire dans ce type de situation hautement enzootique. Quoiqu'il en soit, cet examen a permis d'identifier les espèces présentes ainsi que leur émergence parasitémique relative (élevée pour $T$. congolense, moyenne pour $T$. vivax et très faible pour T. brucei). Associé à la valeur de l'hématocrite, il a permis d'évaluer le taux d'infections ayant des retentissements cliniques décelables. Au moment de l'enquête, selon les techniques (parasitologie et $P(r), 5$ à 10 p. 100 des animaux ont présenté des parasitémies décelables avec prédominance de $T$. congolense, 12 p. 100 des hématocrites inférieurs à 25 et 15 p. 100 l'un au moins de ces paramètres. Seuls les animaux trypanotolérants semblaient peu affectés, mais ils ne représentaient qu'environ 5 p. 100 de la population échantillonnée.

Sans apporter un diagnostic individuel spécifique d'espèce, la réalisation des trois tests Elisa a permis d'établir avec une très bonne sensibilité le taux de porteurs d'anticorps dirigés contre les trypanosomes pathogènes $(81,7 \pm 2,4$ p. 100$)$. Il a permis aussi d'estimer l'incidence annuelle des trypanosomoses à 52 p. 100 en 1997. L'étude des scores maximums de positivité a indiqué une prédominance nette de $T$. vivax dont la séroprévalence a été évaluée à 79 p. 100 et une séroprévalence élevée de $T$. congolense, estimée à 
24 p. 100. La réalisation simultanée des tests Elisa $T$. vivax et T. congolense et l'interprétation des scores de positivité apparaissent fiables. Ils sont recommandées pour l'estimation de la prévalence de ces deux parasites. Compte tenu de l'existence d'importantes réactions croisées du test Elisa $T$. brucei, il est difficile d'estimer la séroprévalence de ce parasite. Mais sa mise en évidence (Mbc et $\mathrm{Pcr}$ ) et les scores de sérologie en faveur d'infections dont il était responsable indiquaient une prévalence inférieure à 3 p. 100. Des outils de diagnostic plus sensibles et spécifiques sont requis pour une estimation plus précise de l'importance de ce parasite dans les populations bovines. Un examen exhaustif des échantillons de cette enquête par $P c r$ apporterait d'autre part un complément d'information.

L'étude sérologique a révélé des contacts fréquents et réguliers de la population bovine avec les trypanosomes. Elle a confirmé que ces parasites demeurent une préoccupation majeure de l'élevage bovin dans la zone nord du secteur de Sidéradougou. Le taux d'animaux paraissant affectés par des trypanosomoses maladies était estimé à 15 p. 100 au moment de l'enquête, mais le taux d'animaux porteurs de parasites était beaucoup plus élevé. Les infections à $T$. vivax étaient hautement enzootiques, mais les états pathologiques du bétail semblaient davantage liés aux infections par $T$. congolense, dont l'incidence annuelle moyenne a été estimée à 14 p. 100. Les données générées par cette enquête seront intégrées dans le système d'information géographique mis en place dans le cadre de l'Atp Santé-environnement du Cirad-emvt (11) pour une analyse multifactorielle du risque trypanosomien. La relation entre prévalence des trypanosomes chez les glossines et chez le bétail sera particulièrement étudiée.

Sur le plan individuel, l'observation des signes cliniques, la mesure de l'hématocrite et la détection des parasites restent des éléments clés dans l'estimation de l'impact clinique et la décision de traitement.

Sur le plan collectif, l'impact de la trypanosomose est davantage évalué par les techniques hématocrites et parasitologiques. En revanche, son épidémiologie est beaucoup mieux appréhendée par la sérologie qui permet d'établir l'incidence des infections et d'estimer leur prévalence ; en ce sens, la sérologie est un outil majeur pour l'identification des secteurs prioritaires de lutte.

\section{Remerciements}

Les auteurs remercient vivement le Cirad (Atp n ${ }^{\circ}$ 70/96), le Cnrs (Programme interdisciplinaire Environnement, vies et sociétés $n^{\circ}$ 96/6/12) et la Coopération française, qui ont apporté leur soutien à ce travail. Ils remercient également chaleureusement le Dr S.M. Touré, directeur du Cirdes au Burkina Faso et le personnel du centre pour leur appui et leur soutien, ainsi que les services de l'élevage et les éleveurs de la zone pour leur coopération.

\section{BIBLIO GRAPHIE}

1. BAUER, B., AM SLER-DELAFO SSE S., KABORE I., KAMUANGA M. 1999. Improvement of cattle productivity through rapid alleviation of African animal trypanosomosis by integrated disease management practices in the Agropastoral Zone of Yalé, Burkina Faso. Trop. Anim. Health Prod., 31: 89-102.

2. BAUER B., PETRICH-BAUER J., KABORE I., KOUROUMA B. MATTAUSCH M., SOME J., TAMBOURA I., 1988. Epidemiological survey in the pastoral zone of Sideradougou, Burkina Faso. In: Proc. int Symp. on modern Insect Control, Vienna, Austria, 16-20 November 1987. Vienna, Austria, AIEA, p. 139-149.

3. BOCQUENTIN R., VERY P., DUVALLET G., 1990. Cinétique des anticorps après traitement trypanocide chez les bovins infectés expérimentalement ou naturellement. Intérêt épidémiologique. Rev. Elev. M éd. vét. Pays trop., 43: 179-186.

4. CAMUS, E., 1983. Diagnostic de la trypanosomose bovine sur le terrain par la méthode de centrifugation hématocrite. Rev. sci. tech. 0 ff. int. Epiz. 2 : 751-769.

5. COCHRAN W.G., 1977. Sampling techniques. New York, NY, USA, Wiley, $448 p$.

6. CONNOR R.J., HALLIWELL R.W., 1987. Bovine trypanosomiasis in Southern Tanzania: parasitological and serological survey of prevalence. Trop. Anim. Health Prod., 19: 165-172.

7. CROSS G.A.M., 1977. Antigenic variation in trypanosomes. Amer. J. trop. Med. Hyg., 26: 240-244.

8. CUISANCE D., POLITZAR H., TAMBOURA I., MEROT P., LAMARQ UE G., 1984. Répartition des glossines dans la zone pastorale d'accueil de Sidéradougou, Burkina Faso. Rev. Elev. Méd. vét. Pays trop., $37:$ 99-113.

9. CUISANCE D., POLITZAR H., MEROT P., TAMBOURA I., 1984. LeS lâchers de mâles irradiés dans la campagne de lutte intégrée contre les glossines dans la zone pastorale de Sidéradougou, Burkina Faso. Rev. Elev. M éd. vét. Pays trop., 37 : 449-468.
10. DE LA ROCQUE S., 1997. Identification des facteurs discriminants majeurs de la présence de glossines dans une zone agropastorale du Burkina Faso. Intérêt pour la prévision du risque trypanosomien. Thèse D oct., U niversité M ontpellier II, M ontpellier, France, 212 p.

11. DE LA ROCQUE S., CUISANCE D., 1997. Facteurs discriminants de la présence de glossines au Burkina Faso. In : Proc. 8th Int. Symp. on Veterinary Epidemiology and Economics, Paris, France, 7-10 juillet 1997. Epidémiol. Santé anim., $n^{\circ}$ spécial : 31-32.

12. DE LA ROCQUE S., LEFRANCOIS T., REIFENBERG J.M., SO LANO P., KABOR I., BENGALY Z., AUGUSSEAU X., CUISANCE D., 1998. $P C R$ analysis ans spacial repartition of trypanosomes infecting tsetse flies in Sidéradougou area (Burkina Faso). Ann. N.Y. Acad. Sc., 849: 32-38.

13. DE LA ROCQUE S., MICHEL J.F., MICHEL V., SOLANO P., DE WISPELAERE G., ARNAUD M., CUISAN CE D., sous presse. Du système pathogène à l'évaluation du risque; cas des trypanosomoses animales dans une zone du Burkina Faso. 25th ISCTRC Meeting, Mombasa, Kenya, 25 sept. - 1 oct.1999. M onbasa, Kenya, O U A/CSTR, 6 p.

14. DESQ U ESNES M., 1997. Standardisation internationale et régionale des épreuves immuno-enzymatiques : méthodes, intérêts et limites. Rev. sci. tech. O ff. int. Epiz., $16: 809-823$.

15. DESQ U ESN ES M., 1997. Les trypanosomoses du bétail en Amérique Latine, étude spéciale dans le Plateau des Guyanes. Thèse Doct., Université Lille, Lille, France, 409 p.

16. DESQ U ESN ES M., TRESSE L., 1999. U tilisation of T. evansi antigens in indirect-ELISA for diagnosis of Chagas disease in humans. In: Proc. of 1st Symposium on New W orld Trypanosomes, 20-22 November 1996, Georgetown, Guyana. Georgetown, Guyana, Vokaty S., Desquenes M. eds, p. 111-114.

17. FERENC S.A., STOPINSKI V., COURTNEY C.H., 1990. The development of an enzyme-linked immunosorbent assay for Trypanosoma vivax and its use in a seroepidemiological survey in the eastern Caribbean basin. Int. J. Parasitol., 20: 51-56.

18. GARDINER P.R., 1989. Recent studies of the biology of Trypanosoma vivax. Adv. Parasitol., 28: 229-317. 
19. GREINER M.. SHIVARAMA BHAT T., PATZELT R. . . KAKAIRE D., SCHARES G., DIETZ E., BÖHNING D., ZESSIN K.H., MEHLITZ D., 1997. Impact of biological factors on the interpretation of bovine trypanosomosis serology. Prev. Vet. Med., 30: 61-73.

20. GUIDOT G., RO ELANTS G.E., 1982. Sensibilité de taurins Baoulé et de zébus à Trypanosoma (Duttonella) vivax et T. (Nannomonas) congolense. Revue Elev. Méd. vét. Pays trop., 35 : 233-244.

21. HOARE C.A., 1972. The trypanosomes of mammals. A Zoological M onograph. O xford, U.K., Blackwell Scientific Publications, 749 p.

22. HOPKINS J.S., CHITAMBO H., MACHILA N., LUCKINS A.G., RAE P.F., VAN DEN BOSSCHE P., EISLER M.C., 1998. Adaptation and validation of antibody-ELISA using dried blood spots on filter paper for epidemiological surveys of tsetse-transmitted trypanosomosis in cattle. Prev. Vet. Med., 37: 91-99.

23. IJAGBONE I.F., STAACK C., REINHARD R., 1989. Fractionation of trypanosome antigens for species-specific sero-diagnosis. Vet. Parasitol., 32: 293-299.

24. LATIF B.M., ADAM K.M., 1973. Differentiation of Trypanosoma brucei, $T$. rhodesiense, and $T$. gambiense by the indirect fluorescent antibody test. Bull World Health O rgan., 48: 401-407.

25. LUCKINS A.G., 1977. Detection of antibodies in trypanosomeinfected cattle by means of a microplate enzyme-linked immunosorbent assay. Trop. Anim. Health Prod., 9: 53-62.

26. LUCKINS A.G., MELHLITZ D., 1978. Evaluation of an indirect fluorescent antibody test, enzyme-linked immunosorbent assay and quantification of immunoglobulins in the diagnosis of bovine trypanosomiasis. Trop. Anim. Health Prod., 10: 149-159.

27. MASIGA D.K., SMYTH A.J., HAYES P., BROMIDGE T.J., GIBSON W.C., 1992. Sensitive detection of trypanosomes in tsetse flies by DNA amplification. Int. J. Parasitol., 22: 909-918.

28. MEROT P., POLITZAR H., TAMBOURA I., CUISANCE D., 1984. Résultats d'une campagne de lutte contre les glossines riveraines au Burkina par l'emploi d'écrans imprégnés de deltaméthrine. Rev. Elev. M éd. vét. Pays trop., 37 : 175-184.
29. MICHEL J.-F., MICHEL V., DE LA ROCQUE S., TOURE I., RICHARD D., 1999. Modélisation de l'occupation de l'espace par les bovins, applications à l'épidémiologie des trypanosomoses animales. Rev. Elev. Méd. vét. Pays trop., 52 : 25-33.

30. MONZON C.M., COLMAN O.L.R., 1988. Estudio seroepidemiológico de la tripanosomiasis equina' (O Mal de Caderas) mediante la prueba de immunofluorescencia indirecta en la Provincia de Formosa (Argentina). Arq. Bras. Med. Vet. Zoot., 40: 279-285.

31. MURRAY M., MURRAY P.K., M CINTYRE W.I.M., 1977. An improved parasitological technique for the diagnosis of African trypanosomiasis. Trans. R. Soc. Trop. Med. Hyg., 71: 325-326.

32. POLITZAR H., CUISANCE D., 1984. An integrated campaign against riverine tsetse, Glossina palpalis gambiensis and Glossina tachinoides, by trapping, and the release of sterile males. Insect Sci. Appl., 5: 439-442

33. ROSSI M., ASO P., ESPINOZA E., 1993. Antigenos de Trypanosoma evansi y su utilización en el immunodiagnostico de la tripanosomiasis bovina. Acta Cient. Venez., 44: 1.

34. SILAYO R.S., GRAY A.R., LU CKINS A.G., 1980. U se of antigens of culturedTrypanosoma brucei in tests for bovine trypanosomiasis. Trop. Anim. Health Prod., 12: 127-131.

35. SO LANO P., MICHEL J.F., LEFRANCOIS T., DE LA ROCQUE S., SIDIBE I., ZOUNGRANA A., CUISANCE, D. 1999. Polymerase Chain Reaction as a diagnosis tool for detecting trypanosomes in naturally infected cattle in Burkina Faso. Vet. Parasitol., 86: 95-103.

36. TOURE S.M., 1977. Diagnostic des trypanosomiases animales. Rev. Elev. M éd. vét. Pays trop., $30: 1-10$.

37. UILENBERG G., 1998. A field guide for the diagnosis treatment and prevention of African animal trypanosomosis. Rome, Italie, FAO, $158 \mathrm{p}$.

38. WHITELAW D.D., GARDINER P.R., MURRAY M., 1988 Extravascular foci of Trypanosoma vivax in goats: the central nervous system and aqueous humor of the eye as potential sources of relapse infections after chemotherapy. Parasitol., 97: 51-61.

Reçu le 14.9.99, accepté le 25.4.00 